\title{
A Ligand-Based Approach to Lead Optimization of N, N'- Substituited Diamines for Leishmanicidal Activity
}

\author{
Charles O. Nnadi 1,*(D), Thecla O. Ayoka ${ }^{2}$ (D) Ndidiamaka H. Okorie $^{3(D)}$ \\ 1 Department of Pharmaceutical and Medicinal Chemistry, University of Nigeria Nsukka, Enugu State Nigeria; \\ charles.nnadi@unn.edu.ng (C.O.N.); \\ 2 Department of Science Laboratory Technology (Biochemistry Unit), Faculty of Physical Science, University of Nigeria, \\ Nsukka, Enugu State Nigeria; thecla.ayoka@unn.edu.ng (T.O.A.); \\ 3 Department of Pharmaceutical and Medicinal Chemistry, Enugu State University of Science and Technology, Enugu State \\ Nigeria; hannah.okorie@esut.edu.ng (N.H.O.); \\ * Correspondence: charles.nnadi@unn.edu.ng (C.O.N);
}

Scopus Author ID 42962136300

Received: 5.10.2021; Revised: 5.11.2021; Accepted: 8.11.2021; Published: 25.11.2021

\begin{abstract}
Several $N, N$-substituted diamines such as putrescine and $N$-monoalkylated derivatives have demonstrated potential as lead compounds against Leishmania donovani at submicromolar levels. There is a need to refine available diamines for enhanced leishmanicidal activity. A 3D-QSAR by Comparative molecular field analysis (CoMFA) on a series of tested diamines for their activities against L. donovani was conducted to understand better the mechanism of action and SARs of the compounds. The model was constructed with AM1 energy minimized conformers of the training set compounds $(n=20)$ by the PLS algorithm method, cross-validated by the method of leave-one-out (LOO), and externally validated using the test set compounds $(n=5)$. A robust model with high predictability of untested compounds was obtained for 2PC (latent variables). The coefficients of determinations for PLS regression $\mathrm{R}^{2}$, internal cross-validation, $\mathrm{Q}^{2}$ and external prediction $\mathrm{P}^{2}$ were 0.97 (SDEC=0.095), 0.82 $(\mathrm{SDEP}=0.102)$ and $0.73(\mathrm{SDEP}=0.115)$ respectively with F-value 618.8 for $2 \mathrm{PC}$. The model coefficients graphically translated into contour maps showed regions where steric $(62 \%)$ and electrostatic $(38 \%)$ properties influence the leishmanicidal activity of the compounds. In addition to the optimum chain length $(n=4)$, a steric effect at position 4 alone or combined with the electrostatic effect at position 3 of the diamine backbone significantly enhanced the leishmanicidal activity. It could further be explored for even higher activity. The model supported the empirical data, which identified $N, N$-substituted diamine as the scaffold for leishmanicidal activities and further provided insights for further optimization of the lead compound.
\end{abstract}

Keywords: CoMFA; diamines; drug design; leishmaniasis; ligand; QSAR.

(C) 2021 by the authors. This article is an open-access article distributed under the terms and conditions of the Creative Commons Attribution (CC BY) license (https://creativecommons.org/licenses/by/4.0/).

\section{Introduction}

Leishmaniasis is a neglected tropical vector-borne disease of the protozoan parasites. Globally, millions of people living in developed and developing regions are infected by 20 different species of Leishmania [1]. The disease is endemic in about 88 countries, with over one billion people currently at risk of infection [2]. It is ranked second to malaria in mortality among protozoan diseases, with incidences of over 1 million cases per annum for both visceral and cutaneous leishmaniasis. Generally, cutaneous leishmaniasis may be less severe and selflimiting compared with the more severe and fatal visceral leishmaniasis. The management of leishmaniasis still depends on chemotherapy with miltefosine, amphotericin B, paromomycin, 
and pentavalent antimonials since effective vaccines for leishmaniasis are still undergoing clinical trials [3]. However, the effective use of these drugs is limited by their side effects, toxicities, cost, low efficacy, and emerging resistance [4]. It is, therefore, imperative to design new effective leishmanicidal agents relying on the many potential drug targets in Leishmania species.

Many enzyme targets such as fructose-1, 6-bisphosphate aldolase, squalene synthase, glyceraldehydes-3-phosphate dehydrogenase, sterol methyltransferase, phosphoglycerate kinase, have been biochemically characterized as potential drug targets for leishmaniasis [5-8]. Identification of these targets represents the rate-limiting step in lead compound optimization [9]. Some of the characterized enzymes are not assayable or available for high throughput screening, rendering them unemployable drug targets [1, 5, 10-14]. Against the backdrop of these challenges associated with target-based optimization of molecules for leishmanicidal agents, we explored the use of a ligand-based approach to a series of $N, N$-substituted diamines for further development and understanding of the mechanism of leishmanicidal activity. In a previous study, the leishmanicidal profile of twenty-five $N, N$-substituted diamines (1-25) synthesized by controlled reductive amination of aliphatic diamines using substituted benzaldehyde was evaluated for in-vitro activity against L. donovani [15].

In this study, the applicability of CoMFA to the optimization of this series of leishmanicidal compounds lies in the congruency of the dataset as well as the congenericity of the diamines with a relatively wide range of potency (3.5 log units). Therefore, we report a 3D-QSAR study on a series of $N, N$-substituted diamines aimed at capturing all the relevant SAR of the diamines to lead modification, (semi-) synthesis design, leishmanicidal activity prediction, understanding the single target-mediated mechanism of activity, and lead optimization of leishmanicidal compound(s).

\section{Materials and Methods}

\subsection{Data set.}

The data set used for the 3D-QSAR study comprises a library of 25 different N.Nsubstituted diamines suggested to interfere with polyamine metabolism of L. donovani (Table 1) and curated from previously synthesized leishmanicidal ligands [15-17]. The library was randomly divided into training $(\mathrm{n}=20)$ and test $(\mathrm{n}=5)$ sets, and the molar $\mathrm{IC}_{50}$ was converted into $\mathrm{pIC}_{50}$ as the target or dependent variable. The $2 \mathrm{D}$ structures of the $N, N$-substituted diamines scaffold, the various substituents, and the $\mathrm{pIC}_{50}$ of the compounds used in the study are shown in Table 1.

\subsection{Pretreatment of data set for the molecular model.}

The 3D structures (1-25) were built in a molecular operating environment (MOE) from the in-built fragments and subsequently energy-minimized by MMFF94x force field followed by low-mode conformational search using default settings of MOE [18]. The resulting best conformers $(\mathrm{dE}=0)$ were further energy-minimized using semi-empirical AM1 Hamiltonian (MOPAC) and the lowest AM1 energy conformers used for the 3D-QSAR study. 


\subsection{Alignment of ligands for modeling.}

The alignment procedure is the most critical step in 3D-QSAR studies. All the 3D structures were manually aligned on compound $12\left(\mathrm{IC}_{50}=0.031 \mu \mathrm{M}, \mathrm{pIC}_{50}=7.5\right)$ as the template following the biophore hypothesis and based on a query of similarity between other compounds and selected atoms/bonds of 12. The template's selected atoms and bonds (Figure 1A) served as a superposition match for the alignment. The superposed molecules together with the $\mathrm{pIC}_{50}$ values were converted to structure data file format for the 3D-QSAR study.

\subsection{Pretreatment of data for CoMFA study.}

The CoMFA study was performed with Open3DQSAR open-sourced software [19]. The aligned molecule assembly was automatically set in a grid box size of $5 \AA$ higher than the largest ligand in both $\mathrm{x}$ - and $\mathrm{y}$ - axes and molecular field grids of $1.0 \AA$ mesh step size. Both Lennard-Jones (Steric) and Coulombic (electrostatic) molecular interaction fields (MIFs) potentials were computed with Open3DQSAR using the MMFF94x Van der Waals parameters and charges. An $\mathrm{sp}^{3}$ carbon atom was used as a probe to compute the steric interaction field, while a volume-less probe with a charge of +1 was used to compute the electrostatic interaction. The pre-filtration of the training set MIF data was done by setting energy (Van der Waals and electrostatics) cutoff at $\pm 30 \mathrm{kcal} / \mathrm{mol}$. In addition, all the variables with standard deviation (SD) $<2.0$ were eliminated to reduce noise and improve the regression analysis [20]. To give the steric and electrostatic fields the same contribution in the PLS model, a block unscaled weighting algorithm was applied to the MIFs.

\subsection{Regression analysis and model validation.}

The regression analysis was performed using the partial least square (PLS) method to the correlation of MIFs with the $\mathrm{pIC}_{50}$ by latent variable (PLS component, PCs) extraction. Smart region definition was performed to improve the model by reducing the dependency of the aligned ligand from grid-to-molecule reciprocal orientations [21]. Internal validation of the model was performed by the leave-one-out (LOO) method for the 5 PCs and expressed as the coefficient of determination $\left(\mathrm{Q}^{2}\right)$ for the correlation of predicted and empirical $\mathrm{pIC}_{50}$ of the 20 ligands used as the training set. The model's predictive ability was determined by predicting the $\mathrm{pIC}_{50}$ of the 5 ligands used as the test set and expressed as the coefficient of determination $\left(\mathrm{P}^{2}\right)$ for correlating predicted and experimental $\mathrm{pIC}_{50}$ data of test set ligands. The optimum PC was selected based on the $\mathrm{Q}^{2}$ obtained for a unit change in constituent. A PC of 2 was selected for this study (Table 2).

\subsection{Contour mapping for Lennard-Jones and Coulombic potential.}

The ReadMOEGRID module of MOE was used in visualization as 3D contour maps of the results of the CoMFA model. The contour maps representing the regression coefficients obtained from the Open3DQSAR were plotted with interest on the steric and electrostatic effects in the structural features of the different ligands of the training set lead to the most significant increase or decrease in leishmanicidal activities [19]. The positive and negative influences of electrostatic interactions were denoted by blue and red contours, respectively, while those of steric interaction on activity was represented by green and yellow contours, respectively 


\section{Results and Discussion}

\subsection{Alignment of ligands and modeling}

The $2 \mathrm{D}$ structures and activity $\left(\mathrm{IC}_{50}\right.$ and $\left.\mathrm{pIC}_{50}\right)$ of the ligands are presented in Table 1. The ligands were classified into a training set for building the model and a test set for external validation. The biophore skeleton used for the alignment is shown in Figure 1A. The best conformer of 12 was used as a template for atom-atom alignment, as shown in Figure 1B.

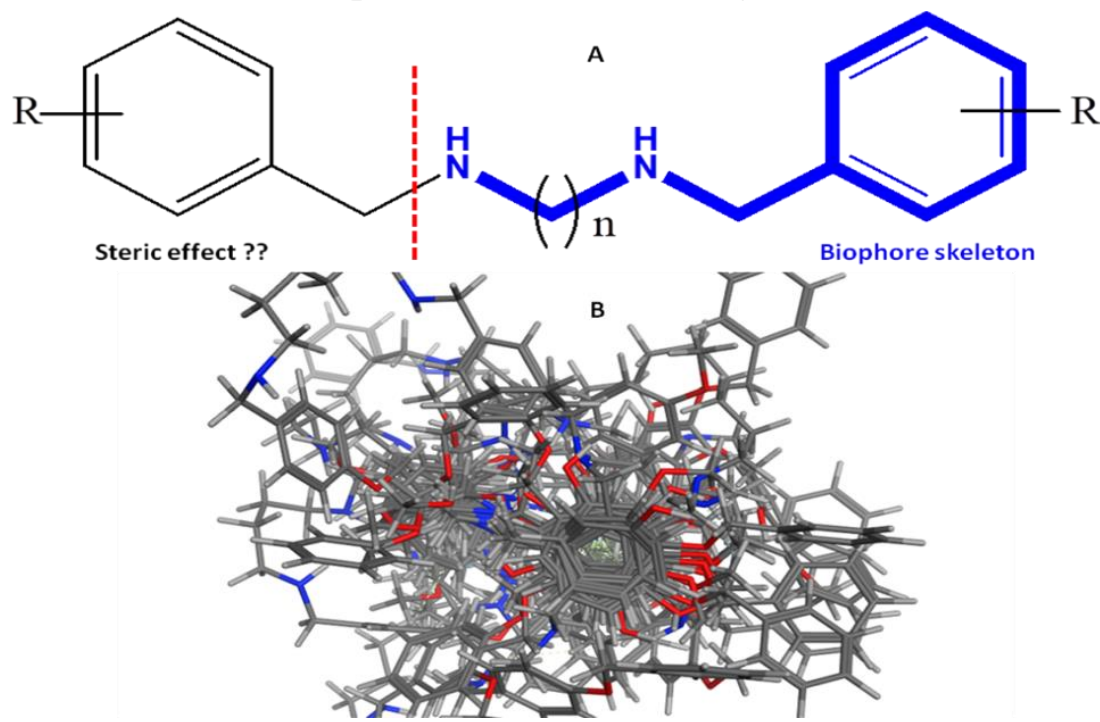

Figure 1. (A) 2D skeleton of ligand used as alignment template (biophore skeleton in blue); (B) Superposed 3D ligands of all $\mathrm{N}, \mathrm{N}$-substituted diamines.

Table 1. 2D structures and activity of ligands.

\begin{tabular}{|c|c|c|c|c|c|c|c|c|c|}
\hline Ligands & $\mathbf{n}$ & $\mathbf{R}$ & $\mathrm{IC}_{50}(\boldsymbol{\mu m})$ & $\mathrm{pIC}_{50}$ & Ligands & $\mathbf{n}$ & $\mathbf{R}$ & $\mathrm{IC}_{50}(\mu \mathrm{m})$ & $\mathrm{pIC}_{50}$ \\
\hline $1 *$ & 3 & $\mathrm{H}$ & 11.01 & 4.958 & 14 & 8 & $4-\mathrm{OBn}$ & 1.14 & 5.943 \\
\hline 2 & 4 & $\mathrm{H}$ & 18.63 & 4.729 & 15 & 10 & 4-OBn & 4.60 & 5.337 \\
\hline 3 & 6 & $\mathrm{H}$ & 13.83 & 4.859 & 16 & 3 & 3-OH, 4-OMe & 100 & 4.000 \\
\hline 4 & 8 & $\mathrm{H}$ & 10.17 & 4.992 & 17 & 4 & $3-\mathrm{OH}, 4-\mathrm{OMe}$ & 100 & 4.000 \\
\hline 5 & 10 & $\mathrm{H}$ & 1.75 & 5.756 & 18 & 6 & $3-\mathrm{OH}, 4-\mathrm{OMe}$ & 43.76 & 4.358 \\
\hline 6 & 3 & $4-\mathrm{OMe}$ & 1.24 & 5.906 & $19 *$ & 8 & $3-\mathrm{OH}, 4-\mathrm{OMe}$ & 44.41 & 4.352 \\
\hline $7 *$ & 4 & $4-\mathrm{OMe}$ & 8.52 & 5.069 & 20 & 10 & $3-\mathrm{OH}, 4-\mathrm{OMe}$ & 4.50 & 5.346 \\
\hline 8 & 6 & 4-OMe & 6.73 & 5.171 & 21 & 3 & 3-OMe, 4-OBn & 0.23 & 6.638 \\
\hline 9 & 8 & 4-OMe & 1.56 & 5.806 & 22 & 4 & 3-OMe, 4-OBn & 0.24 & 6.619 \\
\hline 10 & 10 & 4-OMe & 1.45 & 5.838 & 23 & 6 & 3-OMe, 4-OBn & 0.21 & 6.677 \\
\hline 11 & 3 & 4-OBn & 0.26 & 6.585 & 24 & 8 & 3-OMe, 4-OBn & 0.22 & 6.657 \\
\hline 12 & 4 & $4-\mathrm{OBn}$ & 0.03 & 7.508 & $25^{*}$ & 10 & 3-OMe, 4-OBn & 1.20 & 5.920 \\
\hline $13 *$ & 6 & $4-\mathrm{OBn}$ & 0.24 & 6.619 & & & & & \\
\hline
\end{tabular}

\subsection{CoMFA model statistics and PLS.}

PLS regression with 5PCs was performed to produce a model of a linear relationship between the changes in leishmanicidal activity with the difference in ligands' MIF energies. The model statistics are summarized in Table 2. The best model obtained comprised an optimum number of two latent variables (2PCs) with PLS regression coefficients of determination, $\mathrm{R}^{2}=0.97, \mathrm{Q}^{2}=0.82$, and $\mathrm{P}^{2}=0.73$ for the model calibration, $\mathrm{LOO}$ internal crossvalidation, and the external predictions of the test set ligands' activity (Table 2). The scatter plots of predicted versus experimental pIC50 for the calibration, internal validation, and external prediction are shown in Figure 2. 
Table 2. CoMFA model statistics for leishmanicidal activity (2PCs).

\begin{tabular}{l|l} 
Model parameters & Model statistics, 2PC \\
\hline$R^{2} \pm$ SDEC & $0.970 \pm 0.095$ \\
\hline$Q^{2} \pm$ SDEP & $0.825 \pm 0.102$ \\
\hline$P^{2} \pm$ SDEP & $0.732 \pm 0.115$ \\
\hline$F$-ratio & $618.811(3.895)$ \\
\hline Regression equation & $\mathrm{y}=\mathrm{x}+4.102 \times 10^{-5}$
\end{tabular}

$\mathrm{F}=$ Fisher value (critical $F$-value for the $95 \%$ probability level); SDEC = standard deviation error in calculation; $\mathrm{SDEP}=$ standard deviation error in prediction; $\mathrm{x}=$ actual $\mathrm{pIC}_{50} ; \mathrm{y}=$ predicted $\mathrm{pIC}_{50}$.

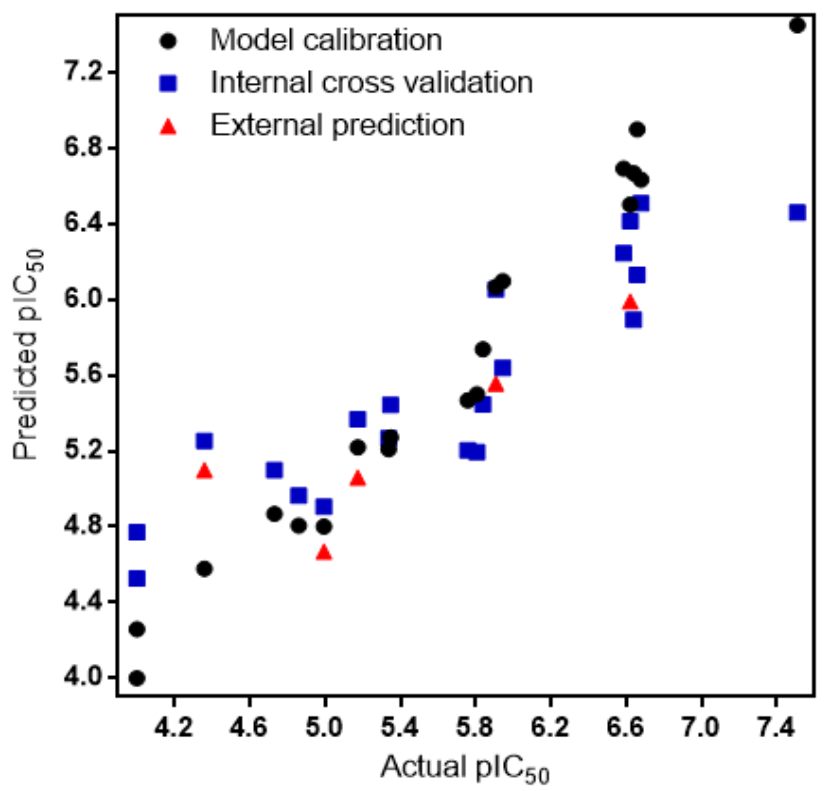

Figure 2. Scatter plot of predicted versus actual leishmanicidal activity (Data obtained from PLS model with 2PC of non-cross validated (black), LOO internal cross-validation (blue), and test set external prediction (red).

\subsection{Analysis of CoMFA contour maps.}

The regression coefficient model for the ligands was translated into contour maps for electrostatic and steric effects on leishmanicidal activity (Figure 3). The contours depict attributes of hypothetical common receptor binding sites where changes of steric and electrostatic properties of the ligands most significantly the ligands' leishmanicidal activity and further provide insight into common receptor-binding areas.

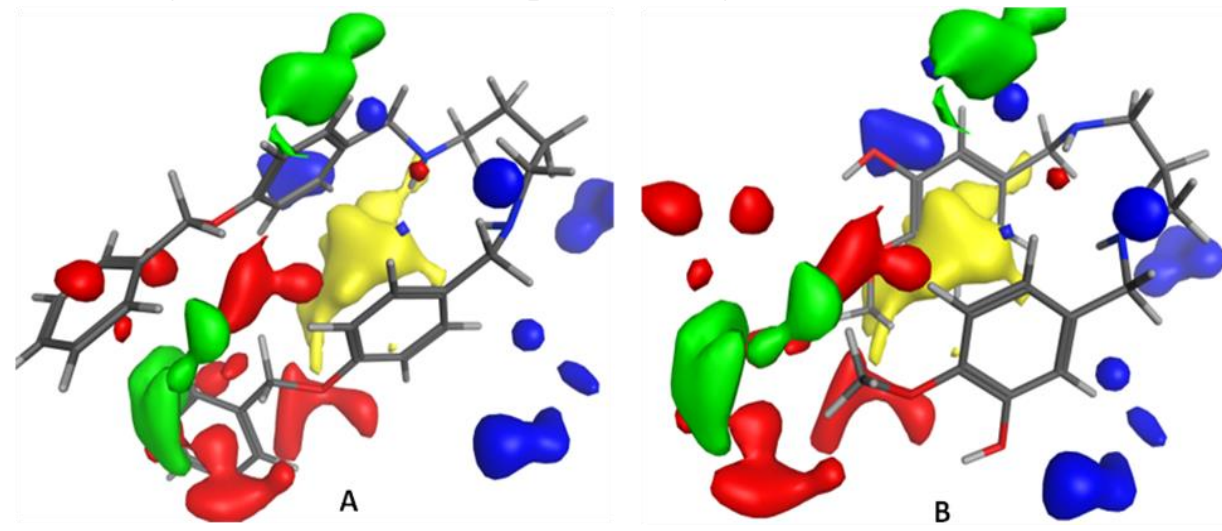

Figure 3. Contour maps showing the CoMFA model for leishmanicidal activity. Ligands shown are the (A) most (12) and (B) least (16) active in the series. Green and yellow regions indicate areas where steric interactions increase and decrease activity, respectively. Blue and red regions represent increased and decreased electrostatic effects with the positively charged probe, respectively. 
The CoMFA model for leishmanicidal activity showed strong PLS correlation with $\mathrm{R}^{2}$ $=0.97, \mathrm{Q}^{2}=0.82$ and $\mathrm{P}^{2}=0.73$. This model $(\mathrm{PC}=2)$ was selected as the optimum PLS component since there was no further significant increase in the $\mathrm{Q}^{2}$ for higher PCs. The model is characterized by its robustness, reliability, and reproducibility since all the correlation coefficients were higher than 0.50 . More so, the model has a low possibility of chance correlation as a result of a high $F$-value, as well as SDEP $>>\mathrm{P}^{2}$. With only a 0.09 unit difference in $\mathrm{Q}^{2}$ and $\mathrm{P}^{2}$, the robustness of the CoMFA model was further confirmed by the closeness of internal $\left(\mathrm{Q}^{2}\right)$ and external $\left(\mathrm{P}^{2}\right)$ coefficients of determination.

Three major factors were found to significantly influence the steric and electrostatic properties of the CoMFA model- the ligand biophore, the carbon chain length of the ligand (n), and the substitution at positions 3 and/or 4 of the phenyl ring(s). The biophore skeleton (Figure 1A) depicts a hypothetical common receptor binding site where changes of steric and electrostatic properties of the ligands most significantly affect the ligands' leishmanicidal activity and further provide insight into common receptor-binding areas [17]. In this study, one of the two $\mathrm{N}$-substituents was found to provide steric influence on the biophore, resulting in a significantly higher effect (62\%) than the $38 \%$ electrostatic effect leishmanicidal activity of the $N, N$-substituted diamines. The CoMFA contour maps of 12 further provided insights into the influence of the steric effect on leishmanicidal activity. There were crowded green and contour within the vicinity of the phenyl moiety in 12 compared with 16 or 17, which has clouds of yellow contour within and around the same region.

The influence of the length of the carbon chain (n) was prominent in 11-15 (Figure 4), which could be attributed to the flexibility of the $\mathrm{sp}^{3}$ carbon chain sandwiched between the diamine of the ligands. It was evident that smaller chain lengths result in pulling the diamines of the ligand away from the blue contour in the CoMFA model maps leading to decreased electrostatic influence and leishmanicidal activity. As the chain length increased, the diamines were pushed nearer to the blue map, though with no trace of red contour, until an optimum of $\mathrm{n}=4$ beyond which the leishmanicidal activity was found to decline. The leishmanicidal activity was found lowest on both sides of the optimum length of the carbon chain due to the "pull-push" effect of $\mathrm{n}$ on the diamines of the ligands

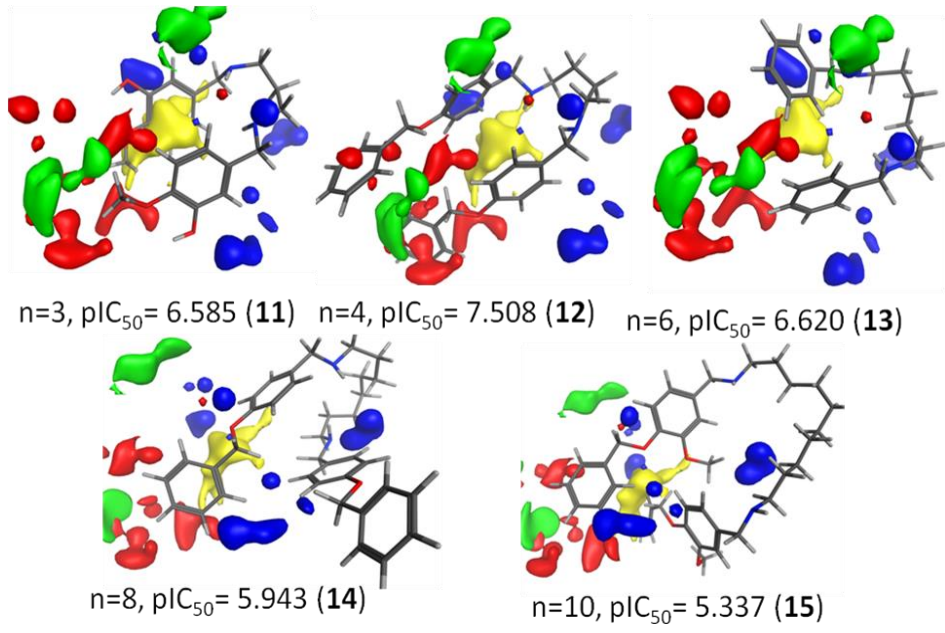

Figure 4. Contour maps showing electrostatic effects on carbon chain length (n).

Another major factor that influenced the leishmanicidal activity of the ligands was the substitution at the $3 / 4$ positions of the phenyl rings. Pairwise comparison of $1 / 6,2 / 7,3 / 8,4 / 9$, and 5/10 showed that 4-Ome substituted ligands increased the electrostatic effects as shown in Figure 5 (circled regions depicted higher densities of the electrostatic effect). A similar effect 
was significant in ligands $1 / 11,2 / 12,3 / 13,4 / 14$, and 5/15, and $1 / 21,2 / 22,3 / 23,4 / 24$, and 5/25. However, 3-OH substituted ligands in 15-20 showed reduced activity earlier attributed to the fact that phenol group increases the ligands' polarity and, therefore, alters that scaffold's ability to cross biomembranes [15, 22, 23]. Except for the increased electrostatic effect due to 3-OMe and 4-OBn observed, this study, however, could not explicitly explain this trend when 15-20 were compared with 21-25.

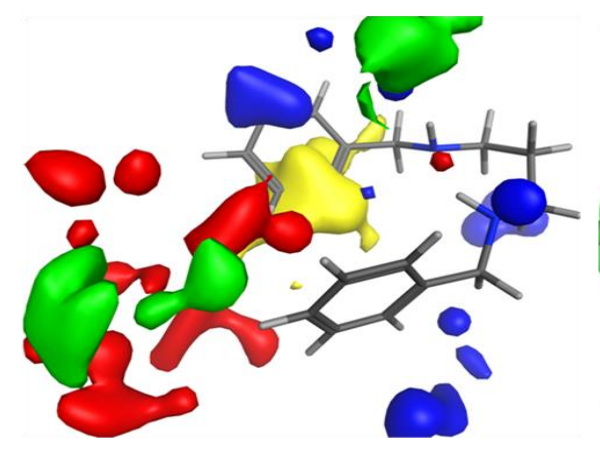

Unsubstituted, $\mathrm{plC}_{50}=4.958(\mathbf{1})$

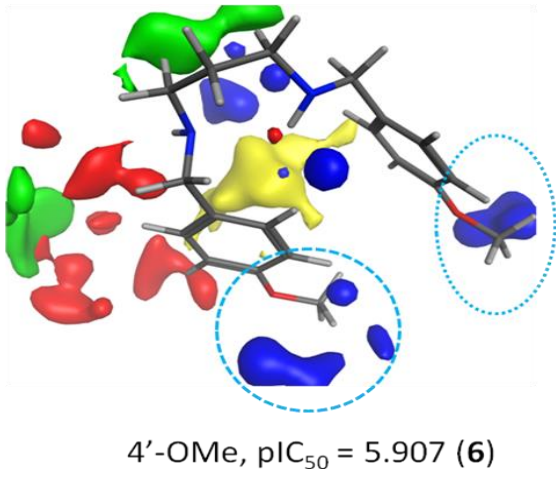

$4^{\prime}-\mathrm{OMe}, \mathrm{plC}_{50}=5.907(6)$

Figure 5. Contour maps showing electrostatic effects on the substitution at the $3 / 4$ positions of the phenyl rings.

\subsection{Application of the model in lead optimization of 12.}

A close examination of the contour maps around the lead compound (12) showed an interesting distribution of the electrostatic contouring (Figure 6). The red contour was prominent at both oxygens of the 4-OBn substituted ligand. This caused a negative electrostatic effect resulting in a lower leishmanicidal activity. Similarly, the second phenyl ring appeared to be surrounded by a yellow contour, suggesting a negative steric effect on the activity. Taken together, the replacement of one or both oxygen(s) of the 4-OBn with $-\mathrm{CH}_{2}$ - could release the negative (red contour) electrostatic influence, thereby potentiating its activity. Alternatively, expunging one of the phenyl-4-OBn from the biophore could remove the negative effects of both the electrostatic and steric effects and subsequently result in a game-changer in leishmaniasis management. This design is currently under evaluation.

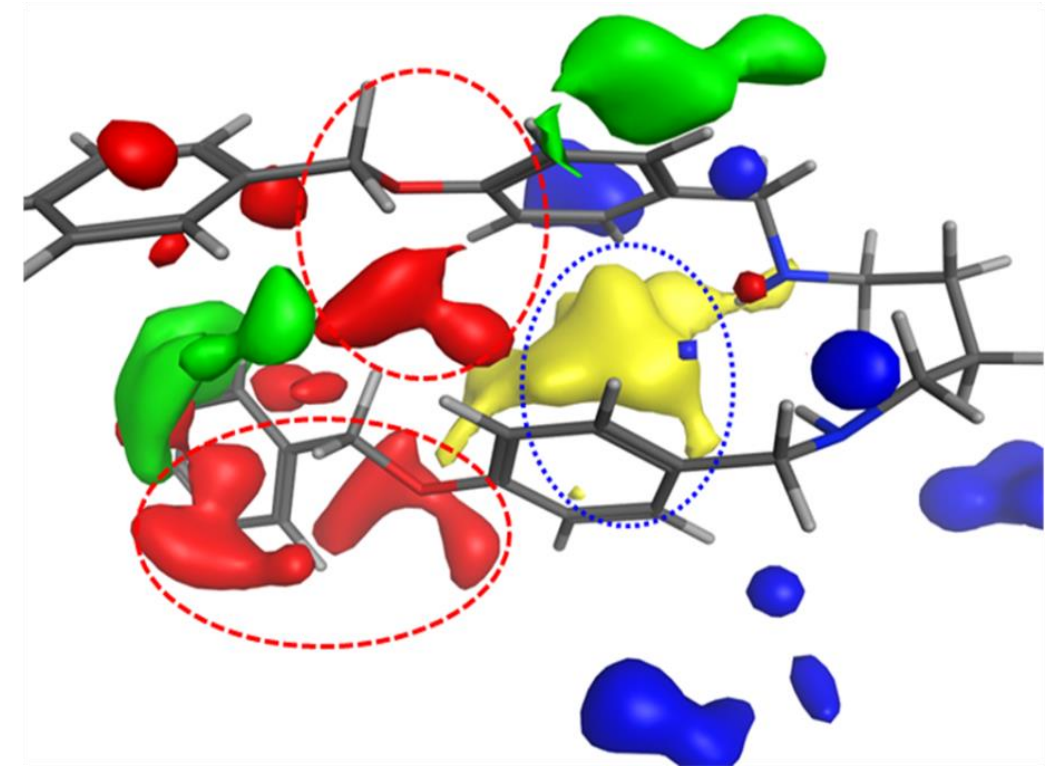

Figure 6. Contour maps showing electrostatic (red circle) and steric (blue circle) effects on the lead compound (12). 


\section{Conclusions}

The CoMFA model obtained in this study was robust, reliable with an insignificant chance correlation. This study identified an $\mathrm{N}, \mathrm{N}$-substituted diamine separated by 4 carbon chains as the optimum biophore for antileishmanial activity. The study also explained the SAR, regression for prediction of the activity of untested ligands, and a starting point for rational drug design of active analogs.

\section{Funding}

This research received no external funding.

\section{Acknowledgments}

The multi-user teaching license for MOE provided to IPBP by the Chemical Computing Group was used for the study

\section{Conflicts of Interest}

The authors declare no conflict of interest.

\section{References}

1. Raj, S.; Sasidharan. S.; Balaji, S.N.; Saudagar, P. An overview of biochemically characterized drug targets in metabolic pathways of Leishmania parasite. Parasitol Res 2020, 119, 2025-2037, https://doi.org/10.1007/s00436-020-06736-X.

2. Lee S.H.; Chaves, M.M.; Kamenyeva, O.; Gazzinelli-Guimaraes, P.H.; Kang, B.; Pessenda, G.; Passelli, K.; Tacchini-Cottier, F.; Kabat, J.; Jacobsen, E.A.; Nutman,T.B.; M2-like, dermal macrophages are maintained via IL-4/CCL24-mediated cooperative interaction with eosinophils in cutaneous leishmaniasis. Science Immunology. 2020, 5, eaaz4415, https://doi.org/10.1126/sciimmunol.aaz4415.

3. Surur, A.S.; Fekadu, A.; Makonnen, E.; Hailu, A. Challenges and opportunities for drug discovery in developing countries: the example of cutaneous leishmaniasis. ACS Medicinal Chemistry Letters. 2020, 11, 12058-2062, https://doi.org/10.1021/acsmedchemlett.0c00446.

4. De Brito, R.C.; de Oliveira Aguiar-Soares, R.D.; de Oliveira Cardoso, J.M.; Coura-Vital, W.; Roatt, B.M.; Reis, A.B. Recent advances and new strategies in Leishmaniasis diagnosis. Applied Microbiology and Biotechnology. 2020, 26, 1-2, https://doi.org/10.1007/s00253-020-10846-y.

5. Raj, S.; Sasidharan, S.; Balaji, S.N.; Saudagar, P. An overview of biochemically characterized drug targets in metabolic pathways of Leishmania parasite. Parasitology Research. 2020, 119, 2025-2037, https://doi.org/10.1007/s00436-020-06736-X.

6. Sasidharan, S.; Saudagar, P. Concerted motion of structure and active site charge is required for tyrosine aminotransferase activity in Leishmania parasite. Spectrochim Acta A Mol Biomol Spectrosc 2020, 232, 118133, https://doi.org/10.1016/j.saa.2020.118133.

7. Tabrez, S.; Rahman, F.; Ali, R.; Alouffi, A.S.; Akand, S.K.; Alshehri, B.M.; Alshammari, F.A.; Alam, A.; Alaidarous, M.A.; Banawas, S.; Dukhyil, A.A. Cynaroside inhibits Leishmania donovani UDPgalactopyranose mutase and induces reactive oxygen species to exert antileishmanial response. Bioscience Reports. 2021, 41, BSR20203857, https://doi.org/10.1042/BSR20203857.

8. Rezende, A.M.; dos Santos,; Vasconcelos, C.R. Systematic in silico evaluation of Leishmania spp. Proteomes for drug discovery. Frontiers in Chemistry. 2021, 9, 228, https://doi.org/10.3389/fchem.2021.607139 .

9. Amiri-Dashatan, N.; Ashrafmansouri, M.; Rezaei-Tavirani, M.; Koushki, M.; Ahmadi, N. Prediction of potential drug targets for cutaneous leishmaniasis by Leishmania major and Leishmania tropica: a quantitative proteomics and bioinformatics approach. Current Science. 2021, 120, 1040-1049, https://doi.org/10.18520/cs/v120/i6/1040-1049.

10. Arya, R.; Dhembla, C.; Makde, R.D.; Sundd, M.; Kundu, S. An overview of the fatty acid biosynthesis in the protozoan parasite Leishmania and its relevance as a drug target against leishmaniasis. Molecular and Biochemical Parasitology. 2021, 21, 111416, https://doi.org/10.1016/j.molbiopara.2021.111416. 
11. Bijlmakers, M.J. Ubiquitination and the Proteasome as Drug Targets in Trypanosomatid Diseases. Frontiers in Chemistry. 2021, 28, 1307, https://doi.org/10.3389/fchem.2020.630888.

12. Bhowmik, D.; Jagadeesan, R.; Rai, P.; Nandi, R.; Gugan, K.; Kumar, D. Evaluation of potential drugs against leishmaniasis targeting catalytic subunit of Leishmania donovani nuclear DNA primase using ligand based virtual screening, docking and molecular dynamics approaches. Journal of Biomolecular Structure and Dynamics. 2021 39, 1838-1352, https://doi.org/10.1080/07391102.2020.1739557.

13. Tabrez, S.; Rahman, F.; Ali, R.; Akand, S.K.; Alaidarous, M.A.; Banawas S, Dukhyil AA, Rub A. Hesperidin Targets Leishmania donovani Sterol C-24 Reductase to Fight against Leishmaniasis. ACS Omega. 2021, 6, 8112-8118, https://doi.org/10.1021/acsomega.0c05858.

14. Tabrez, S.; Rahman, F.; Ali, R.; Akand, S.K.; Alaidarous, M.A.; Alshehri, B.M.; Banawas, S.; Dukhyil, A.A.; Rub, A. Targeting sterol alpha-14 demethylase of Leishmania donovani to fight against leishmaniasis. Journal of Cellular Biochemistry. 2021, 122, 1037-1047, https://doi.org/10.1002/jcb.29922.

15. Caminos, A.P.; Panozzo-Zenere, E.A.; Wilkinson, S.R.; Tekwani, B.L.; Labadie, G.R. Synthesis and antikinetoplastid activity of a series of N,N-substituted diamines. Bioorg Med Chem Lett 2012, 22, 17121715, https://doi.org/10.1016/j.bmcl.2011.12.101.

16. Heby, O.; Persson, L.; Rentala, M. Targeting the polyamine biosynthetic enzymes: A promising approach to therapy of African sleeping sickness, Chagas' disease and leishmaniasis. Amino Acids 2007, 33, 359-366, https://doi.org/10.1007/s00726-007-0537-9.

17. Clark, K.; Niemand, J.; Reeksting, S.; Smit, S.; van Brummelen, A.; Williams, M.; Louw, A.; Birkholtz, L. Functional consequences of perturbing polyamine metabolism in the malaria parasite, Plasmodium falciparum. Amino Acids 2010, 38, 633-644, https://doi.org/10.1007/s00726-009-0424-7.

18. Chemical Computing Group. Molecular Operating Environment (MOE) rel. 2011.10; Chemical Computing Group Inc.: Montreal, QC, Canada, 2014.

19. Tosco, P.; Balle, T. Open3DQSAR: A new open-source software aimed at high throughput chemometric analysis of molecular interaction fields. $J$ Molec Modeling 2011, 17, 201-208, https://doi.org/10.1007/s00894-010-0684-x.

20. Martin, Y.C.; Willett, P.; Heller, S.R. Designing Bioactive Molecules: Three-Dimensional Techniques and Applications (Computer Applications in Chemistry Collection), 1st ed.; American Chemical Society: Washington DC, United States 1998, 181-194.

21. Nnadi, C.O.; Althaus, J.B.; Nwodo, N.J.; Schmidt, T.J. A 3D-QSAR Study on the Antitrypanosomal and Cytotoxic Activities of Steroid Alkaloids by Comparative Molecular Field Analysis. Molecules 2018, 23, 1113; https://doi.org/10.3390/molecules23051113.

22. Wang YL, Wang F, Shi XX, Jia CY, Wu FX, Hao GF, Yang GF. Cloud 3D-QSAR: a web tool for the development of quantitative structure-activity relationship models in drug discovery. Briefings in Bioinformatics. 2021, 22, https://doi.org/10.1093/bib/bbaa276.

23. Hussain W, Majeed A, Akhtar A, Rasool N. Computational studies of 3D-QSAR on a highly active series of naturally occurring nonnucleoside inhibitors of HIV-1 RT (NNRTI). Journal of Computational Biophysics and Chemistry. 2021, 20, 3-11, https://doi.org/10.1142/S2737416520500362. 